\title{
PATTERN OF PRIMARY GLOMERULONEPHRITIS IN DHAKA MEDICAL COLLEGE HOSPITAL, BANGLADESH
}

\author{
PARVEZ IFTEKHAR AHMED ${ }^{1}$, SHAKIB UZ-ZAMAN ${ }^{2}$, FERDOUS JAHAN ${ }^{3}$, RATON DAS GUPTO ${ }^{4}$, \\ MD. NIZAMUDDIN CHOWDHURY ${ }^{5}$, MD. FIROZ KHAN ${ }^{6}$
}

\begin{abstract}
:
Glomerulonephritis are categorised into several different pathological patterns, which are broadly grouped into non-proliferative or proliferative types. ${ }^{4}$ Glomerulonephritis comprises $25-45 \%$ cases of end-stage renal disease (ESRD) in developing nations, like Bangladesh. ${ }^{5}$ This study has been done in Dhaka Medical College Hospital from January 2011 to December 2011 to find out the type of glomerulonephritis among presented glomerulonephritis patients to Inpatient and Outpatient Department of Nephrology aiming to reflect the pattern of glomerulonephritis in Bangladeshi population. The current study duration was 12 months, 128 patients having glomerulonephritis were included in this study. Statistical analysis has been done using the stastical package for social science. In this current study it was observed that most (31.3\%) of the patients were in the age group of 31 to 40 years and the mean $\pm S D$ of age was $32.94 \pm 12.66$ years ranging from 18 to 70 years. In this current study it was observed that $64.8 \%$ was male and $35.2 \%$ was female. Male female ratio was 1.8:1. According to type of glomerulonephtis evaluated by histopathology, it was observed that in Non Proliferative Glomerulonephtis: 12(10.61\%) is Minimal Change Disease (MCD), 9(7.96\%) is Focal Segmental Glomerulosclerosis (FSGS), 25(22.12\%) is Membranous Glomerulonephritis (MGN). In Proliferative Glomerulonephtis: Membranoproliferative Glomerulonephtis- 13(11.5\%), IgA Nephropathy -13(11.5\%), Mesengial Proliferative Glomerulonephtis -14(12.39\%), Focal Segmental Proliferative Glomerulonephtis-14(14.2\%), Rapidly Progressive Glomerulonephtis (RPGN) - 2 (1.8\%). and Chronic Sclerosing Glomerulonephritis is -11(9.7\%). In our study to find out the pattern of Primary Glomerulonephritis in Dhaka Medical College Hospital there is almost similar findings in Bangladeshi and South Asian population which is shown in different studies in this region. We have some variation with Western, European, American, Middle East studies which is may be due to the difference in environmental, genetic, racial, social and economic variation.
\end{abstract}

Key-words: Primary Glomerulonephritis, Renal Biposy, Histological Typing

Received: 17 January 2014

Accepted: 19 June 2014

\section{Introduction:}

Glomerulonephritis (GN) means "inflammation of the glomeruli" and although inflammation is not apparent in all varities (glomerulopathy is sometimes used to denote this), the name sticks. ${ }^{1}$ Biopsy-proven glomerulonephritis (GN) varies in different part of the world due to race, genetic, social, environmental and economic variation. Recent studies suggested a changing pattern of incidence of GN in different parts of the world. ${ }^{2,3}$ They are categorised into several different pathological patterns, which are broadly grouped into non-proliferative or proliferative types. ${ }^{4}$ Histological pattern of glomerulonephritis is important because of treatment modalities. Glomerulonephritis is an important cause of morbidity and mortality among all age group in Bangladesh. Glomerulonephritis comprises $25-45 \%$ cases of endstage renal disease (ESRD) in developing nations,

1. Assistant Professor, Department of Nephrology, Dhaka Medical College Hospital, Dhaka.

2. Assistant Professor, Kidney Foundation, Dhaka, Bangladesh.

3. Medical Officer, Dept. of Medicine, Bangabandu Sheikh Mujib Medical University, Dhaka, Bangladesh.

4. Associate Professor, Department of Nephrology, Dhaka Medical College Hospital, Dhaka.

5. Professor of Nephrology, NIDCH, Mohakhali, Dhaka

6. Professor \& Head, Department of Nephrology, Dhaka Medical College Hospital, Dhaka 
like Bangladesh. ${ }^{5}$ This study has been done in Dhaka Medical College Hospital to find out the type of glomerulonephritis among presented glomerulonephritis patients to Inpatient and Outpatient Department of Nephrology aiming to reflect the pattern of glomerulonephritis in Bangladeshi population.

\section{Material and Methods:}

This study was carried out in the Department of Nephrology, Dhaka Medical College Hospital from January 2011 to December 2011 to find out the type and percentage of Primary Glomerulonephritis in Dhaka Medical College hospital reflecting the pattern of Glomerulonephritis in Bangladeshi population. Patients were selected in all type of glomerulonephritis who had been attended to Nephrology Unit, Dhaka Medical College Hospital. Suspected glomerulonephritis patient was at first diagnosed clinically. Then the patient was diagnosed on the basis of Urinary Heat Coagulation Test, Urinary Assay, 24 hour urinary protein Assay, Urinary Protein Creatinine Ratio and other biochemical tests. Finally the patient was diagnosed histologically and treatment was given and then followed up. For Renal biopsy patient was prepared for biopsy. Patient was investigated for BT, CT, Platelet Count and informed written consent was taken. Two samples from each patient were processed, one for light microscopy and another for immunofluorence microscopy. The spe-cimen for light microscopy preserved in $10 \%$ formalin. The other specimen for direct immunofluorescence (DIF) study, preserved in normal saline. The current study duration was 12 months, 128 patients having glomerulonephritis were included in this study. Inclusion Criteria were all the patients (both sex) with glomerulonephrits in all types, age more than 18 years. Exclution Criteria were Patients with proteinuria other than glomerulonephritis like DM, UTI and Hypertension. After registration of the patient detail history has been taken and physical examination has been done. Patient who had fulfill the inclusion criteria has been enrolled in the study. Detail history, physical examination and investigation has been recorded in a pre designed questionnaire. After completion of collection of data, statistical analysis has been done using the stastical package for social science (SPSS) program for personal computer (ver 11.5). Values has been expressed where necessary as frequency, mean, $\mathrm{SD}$, or on percentage. Prior permission was taken for this study from the Ethical Committee of Dhaka Medical College, Dhaka, Bangladesh, keeping compliance with Helsinki Declaration for Medical Research Involving Human
Subjects 1964, all patients were informed verbally about the study design, the objective, and right for the participant to withdraw from the project at any time, for any reason, what so ever. Written consent was obtained from each subject.

\section{Renal Biopsy}

Renal Biopsy was done in the department of Nephrology, DMCH and tissue was sent for histopathology. Histopathology was done in Armed Forces Institute of Pathology, Dhaka Cantonment, Dhaka.

\section{Observations and Results:}

Table I

Age distribution of the study patients ( $n=128)$

\begin{tabular}{lcc}
\hline Age (in year) & Number of patients & Percentage \\
\hline$<20$ & 26 & 20.3 \\
$21-30$ & 40 & 31.3 \\
$31-40$ & 30 & 23.4 \\
$41-50$ & 22 & 17.2 \\
$51-60$ & 6 & 4.7 \\
$61-70$ & 4 & 3.1 \\
\hline Mean \pm SD & 32.94 & \pm 12.66 \\
Range (min-max) & $(18$ & $-70)$ \\
\hline
\end{tabular}

Above table shows the age distribution of the study patients and observed that, mean \pm SD age was $32.94 \pm 12.66$ years ranging from 18 to 70 years. However, maximum number $40(31.3 \%)$ was found in the age group of 31 to 40 years. Results are depicted in the above table.

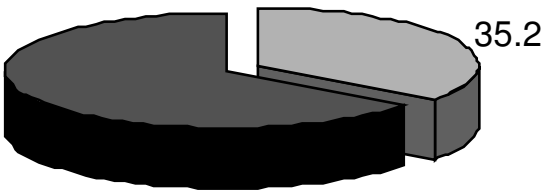

64.8

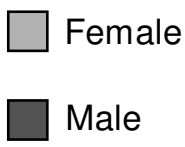

Fig.-I: Sex distribution of the study patients $(n=128)$

Figure I Pie diagram shows the sex distribution of the study patients and observed that, male was $83(64.8 \%)$ which was predominant and female was $45(35.2 \%)$. Male female ratio was $1.8: 1$ in the whole study. Data are shown the pie chart below: 
Table-II

Type of glomerulonephtis on the histopathology. $(n=128)$

\begin{tabular}{lcc}
\hline Type & Number of patients (n=113) & Percentage \\
\hline Non Proliferative glomerulonephtis(46) & & \\
$\quad$ Minimal Change Disease (MCD) & 12 & 10.61 \\
Focal Segmental Glomerulosclerosis (FSGS) & 9 & 7.96 \\
$\quad$ Membranous Glomerulonephritis (MGN) & 25 & 22.12 \\
Proliferative glomerulonephtis (56) & 13 & 11.50 \\
$\quad$ Membranoproliferative Glomerulonephtis & 13 & 11.50 \\
IgA Nephropathy & 14 & 12.39 \\
Mesengial Proliferative Glomerulonephtis & 14 & 12.39 \\
Focal Segmental Proliferative Glomerulonephtis & 2 & 1.80 \\
$\quad$ Rapidly Progressive Glomerulonephtis (RPGN) & 11 & 9.73 \\
Chronic Sclerosing Glomerulonephritis &
\end{tabular}

Inadequate Sample $=15$

According to type of glomerulonephtis evaluated by histopathology, it was observed that in Non Proliferative Glomerulonephtis : $12(10.61 \%)$ is Minimal Change Disease(MCD), $9(7.96 \%)$ is Focal Segmental Glomerulosclerosis (FSGS), 25(22.12\%) is Membranous Glomerulonephritis (MGN). In Proliferative Glomerulonephtis: Membranoproliferative Glomerulonephtis- 13(11.5\%), IgA Nephropathy -13(11.5\%), Mesengial Proliferative Glomerulonephtis -14(12.39\%), Focal Segmental Proliferative Glomerulonephtis-16(14.2\%), Focal Proliferative Glomerulonephtis 14(12.39\%), Rapidly Progressive Glomerulonephtis (RPGN) - 2 (1.8\%). and Chronic Sclerosing Glomerulonephritis is $11(9.7 \%)$.

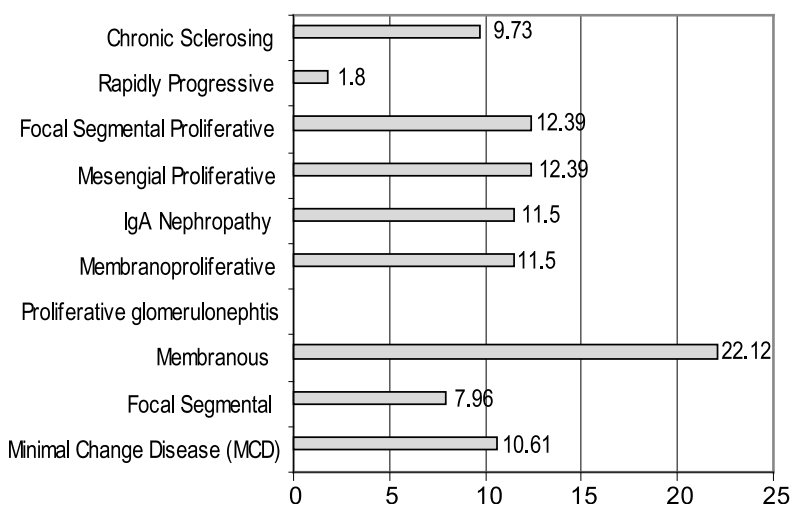

Fig.-2: Bar diagram showing the Percentage of Type of glomerulonephtis on the histopathology.

\section{Discussion:}

This study was carried out with an aim to find out the type and percentage of Glomerulonephritis in Dhaka Medical College Hospital reflecting the pattern of GN in Bangladeshi population. A total number of 128 consecutive patients having glomerulonephritis with 18 to 70 years who came to the Nephrology unit, Dhaka Medical College Hospital, Dhaka during the period of January 2011 to December 2011 were included in this study. Patients with proteinuria other than glomerulonephritis like DM, UTI and Hypertension and Patients under age of 18 were excluded from the study. The present study findings were discussed and compared with previously published relevant studies.

In this current study it was observed that most $(31.3 \%)$ of the patients were in the age group of 31 to 40 years and the mean \pm SD of age was $32.94 \pm 12.66$ years ranging from 18 to 70 years. Rahul,et.al observed almost similar age range and the average age of the patients who underwent the procedure was 34-years. ${ }^{6}$ Hassan , Nima and Alireza observed in their study that the mean \pm SD of patient's age was $31.9 \pm 15.9$ which is also similar to this study. ${ }^{7}$ In another study U Das, KV Dakshinamurty, A Prayaga showed mean age of patients $32.27 \pm 18.37$ (range 10-80) years which is also similar to this study. ${ }^{8}$

In this current study it was observed that $64.8 \%$ was male and $35.2 \%$ was female. Male female ratio was 1.8:1, which indicates that male was predominant in this current study. Rahul,et.al observed there were $139(61.50 \%)$ males and 87 (38.49\%) females, with a 
male to female ratio of $1.5: 1$. which is almost similar to this study. 6 In another study U Das, KV Dakshinamurty, A Prayaga showed in a study The male:female ratio as $1.4: 1$. which is also similar to this study. ${ }^{8}$ But in a study in Rajshahi, Bangladesh Habib and Badruddoza found, 38 (40\%) were males and $57(60 \%)$ were females, with a male to female ratio of $1: 1.5$ which is not similar to this study. ${ }^{2}$

According to type of glomerulonephtis evaluated by histopathology, it was observed that in Non Proliferative Glomerulonephtis is $40.69 \%$, and Proliferative Glomerulonephtisis is $49.58 \%$. In Non Proliferative Glomerulonephtis: $12(10.61 \%)$ is Minimal Change Disease(MCD), 9(7.96\%) is Focal Segmental Glomerulosclerosis (FSGS), 25(22.12\%) is Membranous Glomerulonephritis (MGN). In Proliferative Glomerulonephtis: Membranoproliferative Glomerulonephtis- 13(11.5\%), IgA Nephropathy -13(11.5\%), Mesengial Proliferative Glomerulonephtis -14(12.39\%), Focal Segmental Proliferative Glomerulonephtis 14(12.39\%), Rapidly Progressive Glomerulonephtis (RPGN) - 2 (1.8\%). and Chronic Sclerosing Glomerulonephritis is $11(9.7 \%)$.

According to type of glomerulonephtis evaluated by histopathology, it was observed that in Non Proliferative Glomerulonephtis is $40.69 \%$, Proliferative Glomerulonephtis 49.58\%. Ibrahim and Fayed in a study observed that Proliferative Glomerulonephtis was 497 cases $(53.78 \%)$ and non-proliferative Glomerulonephtis was 427 cases $(46.22 \%)$ which is similar to this study. 9

In this study the most frequent Primary Glomerulonephritis is Membranous Glomerulonephritis $22.12 \%$. Followed by Mesengial Proliferative Glomerulonephtis -14(12.39\%), Focal Segmental Proliferative Glomerulonephtis $14(12.39 \%)$. Jalalah in a study based on retrospective evaluation of archived renal biopsy in the period of 18 years (19892007) showed that the most frequent primary Glomerulonephtis is membranous Glomerulonephtis constituting $25.7 \%$ which is almost similar to this study. But he found focal segmental glomerulosclerosis at $21.3 \%$ where as we found Focal Segmental Glomerulosclerosis $7.96 \%$ which is not similar to that study. He found membrano-proliferative Glomerulonephtis as $11.5 \%$ which is exactly same to this study. ${ }^{10}$

To determine the patterns of glomerulonephritis in Pakistan Rabbani, et.al, studied 511 consecutive renal biopsy reports at the Aga Khan University Hospital during a period of 18 years from January 1990 to December 2008. The most common histological lesion was membranoproliferative disease (28\%). Membranous Glomerulonephtis was the second most common lesion (19\%). The percentage of Membranoproliferative Glomerulonephtis differ from this study which is $13(11.5 \%)$, but the percentage of Membranous Glomerulonephritis $(22.12 \%)$ is almost similar to this study. ${ }^{11}$

In a study Nasir Ahmed, Md. Mohosin, Nurul Huda found that Diffuse Membranoproliferative Glomerulonephritis is $40.90 \%$, Focal Segmental Glomerulosclerosis - 22.72\%, Minimal Change Disease - $18.18 \%$, Membranous Glomerulonephritis9.09\%, IgA Nephropathy $-4.54 \%$. In comparison to this study we have some variation. 12

Habib and Badruddoza studied on 95 adequate renal biopsies done during July 2008 to June 2009 in the Department of Pathology, Rajshahi Medical College, Bangladesh and showed Focal and segmental mesangial proliferative glomerulonephritis was the most common histological lesion in $29.47 \%$. Diffuse mesangial proliferative glomerulonephritis was the second most common lesion (15.79\%), followed by focal segmental glomerulonephritis (11.58\%), minimal change disease (10.53\%), membranous glomerulonephritis (7.37\%), IgA nephropathy (6.85\%), chronic sclerosing glomerulonephritis $(2.11 \%)$ and crescentic glomerulonephritis $(2.11 \%)$. In comparison to this study we have some variation. ${ }^{2}$

In our study to find out the pattern of Primary Glomerulonephritis in Dhaka Medical College Hospital there is almost similar findings in Bangladeshi and South Asian population which is shown in different studies in this region. But there is some variation which may be due to no clear histological typing guideline around the world. We have some variation with Western, European, American, Middle East studies which is may be due to the difference in environmental, genetic, racial, social and economic variation. At the same time we recommend to have a histologic typing guideline for proper management of Glomerulonephritis.

\section{Summary :}

This study was carried out with an aim to find out the type and percentage of Glomerulonephritis in Dhaka Medical College Hospital reflecting the pattern of glomerulonephritis in Bangladeshi population. A total number of 128 consecutive patients having glomerulonephritis with over 18 years who came in the Nephrology unit, Dhaka Medical College Hospital, Dhaka during the period of January 2011 to December 2011 were included in this study. Patients with proteinuria other than glomerulonephritis like DM, 
UTI and Hypertension and Patients under age of 18 were excluded from the study. The present study findings were discussed and compared with previously published relevant studies.

In this current study it was observed that most $(31.3 \%)$ of the patients were in the age group of 31 to 40 years and the mean \pm SD of age was $32.94 \pm 12.66$ years ranging from 18 to 70 years.

In this current study it was observed that $64.8 \%$ was male and $35.2 \%$ was female. Male female ratio was 1.8:1, which indicates that male was predominant in this current study.

According to type of glomerulonephtis evaluated by histopathology, it was observed that in Non Proliferative Glomerulonephtis: $12(10.61 \%)$ is Minimal Change Disease(MCD), 9(7.96\%) is Focal Segmental Glomerulosclerosis (FSGS), 25(22.12\%) is Membranous Glomerulonephritis (MGN). In Proliferative Glomerulonephtis: Membrano-proliferative Glomerulonephtis- 13(11.5\%), IgA Nephropathy 13(11.5\%), Mesengial Proliferative Glomerulonephtis -14(12.39\%), Focal Segmental Proliferative Glomerulonephtis-14(14.2\%), Rapidly Progressive Glomerulonephtis (RPGN) - 2 (1.8\%). and Chronic Sclerosing Glomerulonephritis is $-11(9.7 \%)$.

\section{Conclusions:}

In our study to find out the pattern of Primary Glomerulonephritis in Dhaka Medical College Hospital there is almost similar findings in Bangladeshi and South Asian population which is shown in different studies in this region. We have some variation with Western, European, American, Middle East studies which is may be due to the difference in environmental, genetic, racial, social and economic variation.

\section{References :}

1. Goddard. J., Turner, A.N., Stewart, L.H., 2010 . Kidney and Urinary Tract Disease. In : Colledge, N.R., Walker, B.R., Ralston, S.H. , 2010, Davidson's Principle and Practice of Medicine, $21^{\text {st }}$ edition, page: 500. Publisher: Churchill Livingstone.

2. MA Habib, SM Badruddoza, 2012. Pattern of glomerular diseases among adults in Rajshahi, the
Northern Region of Bangladesh. Saudi J Kidney Dis Transpl. 23 (4), pp. 876-880.

3. Swaminathan S, Leung N, Lager D, 2006 . Changing incidence of glomerular disease in Olmsted county, Minnesota: a 30-year biopsy study. Clin J Am Soc Nephrol, 1, pp: 483-487.

4. Kumar,V., Abbas,A.K. , Cotran,R.S., Aster,J., 2010. The Kidney. Robbins \& Cotran Pathologic Basis of Disease, $8^{\text {th }}$ edition, Publisher: W.B. Saunders Company.

5. Rashid HU, 2003. Nephrotic Syndrome-Evidence Based Management. Bangladesh Renal Journal 22, pp:1-4.

6. Rahul Mannan, Tejinder Singh Bhasin, Pramela Anthony Singh, Vatsala Misra, Mridu Manjari, 2012. The Pattern of Glomerulonephritis in the North Indian Gangetic Plain- A 13-Year Epidemiological Study. Journal of Clinical and Diagnostic Research. 6(5): 855-858.

7. Mohammad Hoseiniakbari Hassan, Rezaei Nima, Rezaei Alireza, 2009. Pattern of glomerulonephritis in Iran: A preliminary study and brief review. Medical science monitor, 15(9), pp:109-114.

8. U Das, KV Dakshinamurty, A Prayaga, 2011. Pattern of biopsy-proven renal disease in a single center of south India: 19 years experience. Indian Journal of Nephrology, 21(4), pp: 250-257.

9. Salwa Ibrahim, Ahmed Fayed, 2009. A five-year analysis of the incidence of glomerulonephritis at Cairo University Hospital-Egypt Clinical Kidney Journal oxfordjournals. 2(5), pp: 431-435.

10. Jalalah SM. , 2009. Patterns of primary glomerular diseases among adults in the western region of Saudi Arabia.. Saudi Journal Kidney Disease Transplant. 20(2), pp: 295-9.

11. Malik Anas Rabbani, Ghulam Murtaza Memon, Bushra Ahmad, Shehla Memon, Syeda Anjala Tahir, Shumaila Tahir , 2012. Percutaneous renal biopsy results: a retrospective analysis of 511 consecutive cases. Saudi Journal Kidney Disease Transplant. 23(3), pp: 614-618.

12. Nasir Ahmed, Md. Mohosin, Nurul Huda, 2009. The Pattern Of Glomerulonephritis in a study of Kidney Biopsy Proven Cases in Mitford Hospital. Bangladesh Renal Journal; 28( 2), pp : 29-31. 